\title{
Pulsation and its rôle for circumstellar features
}

\author{
A.Heske \\ Sterrewacht Leiden \\ P.O.Box 9513 \\ 2300 RA Leiden, The Netherlands
}

Circumstellar envelopes of cool giants and supergiants contain atomic and molecular gas, and dust. The charateristic spectral features of these different components can be observed at optical (atoms), at radio (molecules) and at infrared wavelengths (dust). Since the detection of circumstellar matter around giants and supergiants most studies concentrated on detemining mass loss rates from observations of a single component assuming steady mass loss during late stellar evolution. Nevertheless, the IRAS colour colour diagramme and evolutionary models rather point to a non steady evolution during the mass loss phase of the star.

Little is known how the structure of the circumstellar envelope and the spectral features evolve along the giant branch and by which stellar parameters they are determined.

To investigate these problems a sample of about $100 \mathrm{cool}$ giants and supergiants with spectral types between $\mathrm{KO}$ and $\mathrm{M} 8$, selected by their peculiar infrared colour (I-K), was observed at optical ( $H \alpha$ and KI [potassium]), at infrared $(1-20 \mu m$, including IRAS data) and at radio wavelengths (SiO at $43 \mathrm{GHz}$ and $\mathrm{CO}$ at $115 \mathrm{GHz})$.

1 - A strong correlation was found between $\mathrm{SiO}$ masers, $H \alpha$ emission and multiple absorption components in the KI resonance absorption profile. It seems to hold for giants but not for supergiants. This suggests that shock waves act as a trigger for $\mathrm{SiO}$ maser emission and are responsible for multiple shell structure in envelopes of Mira variables.

2 - The asymmetry of the light curve and the period have significant influence on the strength of the $9.7 \mu \mathrm{m}$ emission feature, i.e. the optical depth of the dust.

3 - The expansion velocities derived from the thermal $\mathrm{SiO}(\mathrm{v}=0)$ line are systematically about $2 \mathrm{~km} / \mathrm{s}$ lower than those derived from the CO line.

This indicates that $\mathrm{SiO}$ emission originates in parts closer to the star where acceleration is still taking place.

These results lead to the conclusion that once pulsation becomes important during the evolution of cool giants the structure of circumstellar envelopes changes drastically. 